Revue d'histoire de l'Amérique française

ANG REVUE D.HISTOIRE DE L'AMÉRIQUE FRANÇAISE

\title{
Le cheval et ses implications historiques dans l'Amérique française
}

\section{Robert-Lionel Séguin}

Volume 5, numéro 2, septembre 1951

URI : https://id.erudit.org/iderudit/801699ar

DOI : https://doi.org/10.7202/801699ar

Aller au sommaire du numéro

Éditeur(s)

Institut d'histoire de l'Amérique française

ISSN

0035-2357 (imprimé)

1492-1383 (numérique)

Découvrir la revue

Citer cet article

Séguin, R.-L. (1951). Le cheval et ses implications historiques dans l'Amérique française. Revue d'histoire de l'Amérique française, 5(2), 227-251.

https://doi.org/10.7202/801699ar d'utilisation que vous pouvez consulter en ligne.

https://apropos.erudit.org/fr/usagers/politique-dutilisation/ 


\section{LE CHEVAL ET SES IMPLICATIONS HISTORIQUES DANS L'AMÉRIQUE FRANÇAISE}

Pour la partie septentrionale de l'Amérique française, un auteur $^{1}$ a fixé la première importation de chevaux à l'sle de Sable au large des côtes acadiennes. Ces bêtes amenées par le baron de Lery en 1518 seraient devenus la souche des chevaux sauvages qui habitèrent cette île jusqu'en 1936, époque où ils sont capturés, au nombre d'une centaine environ, et transportés en Nouvelle-Écosse, d'après une décision du département de la Marine.

Il reste indéniable que des troupeaux de chevaux sauvages se trouvaient à l'île de Sable. Ainsi s'exprime l'arpenteur Bouchette en $1832::^{2}$ "The only native animals to be met with are some wild horses, whose flesh has been occasionnally found a providential substitute for better food."

Mais sur la date de l'arrivée de ces solipèdes, rien n'est moins certain. Dionne cependant est l'un des rares auteurs à prétendre que le baron de Lery aurait déposé des vaches et des chevaux à l'île de Sable $^{3}$. D'autre part rien ne prouve d'une façon indiscutable que de Lery ait eu des chevaux dans les cales de ses vaisseaux. Cartier n'en fait aucune mention dans la narration de ses voyages, quoi qu'il y soit longuement question de l'expédition du gentilhomme calviniste ${ }^{4}$. A ce sujet Lescarbot rapporte ce qui suit ${ }^{5}$ :

mais la longueur du voyage l'ayant trops longtemps tenu sur la mer il (le baron de Lery) fut contraint de décharger là (à l'ile de Sable) son bestail vaches \& pourceaux, faute d'eaux douces \& de paturages.

1. Frère Isidore o.c.r., L'Elevage du Cheval (Oks, 1940), 17.

2. Joseph Bouchette, The British Dominions in North America (2 vol., London 1831-1832) 2: 72 .

3. N.-E. Dionne, La Nouvelle-France de Cartier d Champlain 1540-1609 (Qu6bec, 1891), 174.

4. Bref Recit et Succinete Narration de la Navigation par le capitaine Jacques Cartier aux iles de Canada (3 vol., Paris, Tross, 1866), 3: 7 .

5. Marc Lescarbot, Histoire de la Nouvelle-France, contenant les navigations faites par les Francais es Indes Occidentales et Nouvelle-France souz l'avars et authorite de noe Roys Tres-Chrétiens... (4 vol., Paris, 1866), 1: 20. 
Aussi lorsque le marchand de la Roche enverra chercher les "abandonnés" du poste avancé de l'île de Sable, Lescarbot poursuivra: "ils (les abandonnés) vivaient de poissons et du laictage de quelques vaches qui y furent apportés par le sieur Baron de Leri6". Il n'est pas encore question de chevaux.

D'après le récollet Sixte Le Tac le marquis de La Roche aborda à l'île de Sable en mai 1598, pour trouver: "quesques vaches \& pourceaux qui y avoient été laissés soit par le baron de Lery, soit par les Portugais qui y avoient tenté un établissement ${ }^{7}$."

Plus tard Champlain écrira en date du ler mai $1604^{8}$ :

L'isle (ile au Sable) est fort sablonneuse \& n'y a point de bois de haute futaie, se ne sont que taillis \& herbages que pasturent des bœufz \& vaches que les Portugais y porterent il y a plus de 60 ans, qui servirent beaucoup aux gens du Marquis de la Roche.

Nouvelle version sur l'origine des bêtes à l'île de Sable. Mais encore le cheval est-il passé sous silence.

Mais sortons de la Nouvelle-France. Le 30 mai 1539, De Soto, l'intrépide compagnon de Pizarre, débarque en Floride. Les autochtones lui sont hostiles et s'acharnent surtout contre "ses chevaux de guerre"'. La cavalerie du "conquistador" compte 350 bêtes ${ }^{10}$. Le 1er aout l'explorateur espagnol est au village de Coosa dans l'Etat actuel de l'Alabama. Le chef Tuscabeza monte un cheval que lui a présenté De Soto pour suivre l'expédition ${ }^{11}$. La petite colonne marche sur Mauvila avec la moitié de sa cavalerie. Le chef indien Tuscaloussas et ses hommes ne prisent guère la présence des "visiteurs". Il y a choc le 1er décembre 1540 et les espagnols perdent 82 hommes et

6. Ibid., 20; édition de 1612, 22.

7. Père Sixte LeTac, Histoire chronologique de la Nouvelle France ou Canada, depuis sa découverte (mil cinq cents quatre) jusques en l'an mil six cents trente deux.) (Paris, 1888), 58.

8. Oeuvres de Champlain, édition Laverdière (6 vol. Québec, 1870), 1: 7. Relation de 1604 .

9. Alcée Fortier, A History of Louisiana (4 vol., New-York, 1904), 1: 6. 1: 7 .

10. Charles Gayarré, Histoire de la Louisiane (2 vol., Nouvelle-Orléans, 1846),

11. Alcée Fortier, A History of Louisiana (4 vol., New-York, 1904), 1: 8. 
42 chevaux $^{12}$. Poursuivant leur marche, ils arrivent à l'État actuel du Mississippi dans la contrée des Chickasaws où ils doivent livrer un combat sanglant qui leur coûte 40 hommes et 50 chevaux ${ }^{13}$. De Soto s'empresse de se fortifier et les "vaincus" ne sont pas lents à prendre leur revanche. Vers la fin de janvier 1541, profitant d'un rude vent $\mathrm{du}$ nord, les Indiens attaquent en pleine nuit le village occupé par les Espagnols et allument partout l'incendie. Le gouverneur est le premier "à cheval" pour repousser l'attaque ${ }^{14}$. Il est accompagné de dix ou douze cavaliers. La violence du brasier est telle que les cavaliers n'ont pas eu le temps de s'armer et de seller leurs chevaux. Il y en a qui cherchent à se sauver en traînant leurs bêtes par la bride ${ }^{15}$. Malgré sa bravoure, De Soto ne peut empêcher deux colonnes ennemies d'entrer dans le village et de mettre à mort beaucoup de chevaux. Bientôt le capitaine Vasconcellos vient soutenir son chef avec vingt-quatre cavaliers portugais ${ }^{16}$. Grâce à ce renfort les Indiens dégringolent vers la forêt proche poursuivis par De Soto à la tête de la cavalerie. Le combat a coûté 40 hommes et 50 chevaux aux Espagnols.

Revenons en Nouvelle-France. En avril 1541 un espion espagnol, posté sur la côte bretonne, épie les préparatifs de l'embarquement de Cartier qui se dispose à entreprendre son troisième voyage en Canada. De Saint-Malo "l'agent" envoie un "mémoire" à la cour de Charles-Quint dans lequel nous relevons ce qui suit"

Ilz (Cartier et ses hommes) maynent aussi vingt vaches vives, quatre thoureaulx, cent brebis et moutons, cent chievres, et dix pourceaulx, pour les faire multiplier au pays où ils vont. et avec ce vingt chevaulx et jumentz pour Charrier les choses necessaires a edifier et fortifier.

12. Ibid.

13. Ibid., 9. 10.

14. Charles Gayarré, Histoire de la Louisiane (2 vol., Nouvelle-Orléans, 1846), 1 :

15. Ibid., 11. 1: 12.

16. Charles Gayarré, Histoire de la Louisiane (2 vol., Nouvelle-Orléans, 1846),

17. H.P. Biggar, A Collection oy Documents relating to Jacques Cartier and the Sieur de Roberval (Ottawa, Public Archives of Canada, 1930), 278. Rapport d'un espion espagnol sur les armements de Jacques Cartier. St-Malo, avril 1541. 
Par excès de zèle, l'émissaire espagnol aurait-il surchargé son rapport? Dans la relation de son troisième voyage de 1541, Cartier ne fait aucune mention de la race chevaline. Dans le récit de ses voyages antérieurs, le découvreur se contente de noter que le mercredi 18 aout (1535) il "apperçu plusieurs poissons, qui ont forme de chevaulx , lesquelz vont à terre de nuict \& de iour à la mer"18.

D'autre part, dès 1609 à Port-Royal, aux premières heures de l'Acadie française jusqu'au pillage de la place par Argall en 1613, Lescarbot nous apprend que chaque année "on multiplie la culture et le défrichement. Dans le haut de la rivière on y eleve des poulains, des veaux et des pourceaux"19. Mais le sac de Saint-Sauveur incite Thomas Gale, gouverneur de la Virginie, à confier à Argall une nouvelle expédition qui atteint la rade de Port-Royal à la fin d'octobre 1613. Comme les deux couronnes sont en paix, les Anglais ne sont pas inquiétés et peuvent débarquer dans la place. Biencourt est alors chez les sauvages. Les Anglais rasent le fort et les habitations de Port-Royal et emportent jusqu'aux charpentes de menuiseries après les avoir démontées. Profitant de la marée, ils remontent la rivière Dauphin et enlèvent des chevaux et des poulains qui paissent dans la prairie ${ }^{20}$. Par ailleurs les sieurs Poutrincourt et de Biencourt, père et fils, semblent vouloir transformer Port Royal en colonie de comptoir. C'est ce qui détermine Madame de Guercheville à jeter les bases de la colonie acadienne de Saint-Sauveur. Dans ce dessein on frète un vaisseau qui fait voile de Honfleur le 12 mars 1613. Il doit atteindre l'Acadie le 16 mai suivant. Le Père Biart note que le navire porte dans ses flancs "des chevaux pour le labour des terres"

D'autre part, le 25 aout 1620 le récollet Denis Jamet détaille comme suit le cheptel acadien: "nous avons amené un Asne \& une

18. Bref Recit et Succincte Narration de la Navigation faite en MDXXXV et MDXXXVI par le Capitaine Jacques Cartier aux iles du Canada, Hochelaga, Saguenay et autres. (Paris, Tross, 1863), 122.

19. Marc Lescarbot, Histoire de la Nouvelle-France contenant les navigations faites par les Français ès Indes Occidentales et Nouvelle France souz l'avou et authorite de noz Roys Très-Chrétiens... (4 vol., Paris, 1617). Description du pillage de PortRoyal.

20. Rameau de Saint-Père, Une Colonie fédale en Amerique - L'Acadie (16041881) (2 vol., Paris et Montréal, 1889), 1: 60.

21. [ Abbé E.M. Faillon ], Histoire de la Colonie frangaise en Canada (3 vol., Montréal, 1865-1866), 1: 114. 
Anesse pour notre commodité, nous nourrissons aussi des Pourceaux, un couple d'oyes masle $\&$ femelle, Sept paire de volailles, quatre paires de Cannes. Quand aux vaches \& cheuvres, nous ne sommes pas en volonté d'en nourir que l'année prochaine"22. Il n'est pas encore question des chevaux.

Il en est de même en Nouvelle-France. Mais loin de le déplorer, le Père Le Jeune écrira dans la relation de 1634: "On a cette année, amener quelques anes qui rendent de tres bons services. Les chevaux pourroient servir, mais rien ne presse d'en amener." ${ }^{23}$

Cette dernière observation est bien significative. Comment expliquer ce désintéressement envers les chevaux? D'après Pierre Boucher il aurait été difficile de les nourrir, car la récolte des plantes fourragères est presque nulle ${ }^{24}$. Certes, les prairies peuvent remplir les fenils, mais on ne récolte pas le foin sans danger, tant que les Iroquois font la ravaude, surtout aux habitants de Montréal et des Trois-Rivières; faucheurs et faneurs deviennent des cibles faciles pour les fils des Cinq-Cantons qui, à la façon des couleuvres, se coulent entre les herbes hautes du mil. Une autre raison empêche encore les habitants de s'intéresser aux chevaux. C'est que les frais de transport à bord des vaisseaux de France restent toujours très élevés. Peu de personnes peuvent se payer ce "luxe"; surtout l'on craint qu'une fois arrivés les chevaux ne succombent sous les flèches iroquoises, comme c'est le cas pour les autres bestiaux ${ }^{25}$.

L'on comprend donc que la Métropole ne se montre pas trop "anxieuse" d'introduire le cheval en Nouvelle-France. Depuis les premières années de la colonie laurentienne, les seuls animaux de trait sont les bœufs, les vaches et les ânes. Le 25 juin 1647, le premier

22. F. Gabriel Sagard, Histoire du Canada et voyages que les frères mineurs Recollects $y$ ont faicts pour la conuersion des infidelles, etc. (3 vol., Paris, 1866), 1: 70. Lettre du P. Denis Jamet au sieur des Boues.

23. Relations des Jésuites contenant ce qui s'est passé de plus remarquable dans les missions des Pères de la Compagnie de Jésus dans la Nouvelle-France. Ouvrage publié sous les auspices du gouvernement canadien. (3 vol., Québec, 1858), 1: 45-92.

24. Pierre Boucher, Histoire véritable et naturelle des mours et productions du pays de la Nouvelle-France, (Montréal 1882).

25. Thomas Chapais, Jean Talon, intendant de la Nouvelle-France (1665-1672) Québec, 1904), 291. 
cheval venu de France débarque à Québec. Une note laconique du Journal des Jésuites nous l'apprend:

Ce mesme vaisseau aporta le 1er cheval, dont les habitans faisoient présent à M. le Gouverneur ${ }^{26}$.

Ce cheval est un cadeau offert par les habitants à M. de Montmagny qui a succédé à Champlain comme gouverneur depuis le 12 juin $1636^{27}$. Le nouveau représentant de Sa Majesté s'est fait immédiatement remarquer par sa sagesse et ses grandes qualités. Laissons la parole au jésuite Charlevoix ${ }^{28}$ :

M. de Montmagny, qui succéda à M. de Champlain dans le Gouvernement du Canada, \& M. de Lisle, qui commandoit aux Trois-Rivières, tous deux Chevaliers de Malte, faisoient hautement profession d'une piété, qui convenoit à leur Etat $\&$ montroient pour le bon ordre un zèle dont la fermeté \& leur exactitude assuroient le succès.

Il semble que ce soit surtout à titre de chevalier de l'île de Malte que M. de Montmagny doive son cadeau. Les habitants trouvaient inconvenant qu'un chevalier demeure sans monture. C'est du moins l'opinion de feu Ernest Gagnon: "Les habitants de ce temps-là avaient de l'esprit comme ceux d'aujourd'hui: ils jugeaient qu'un chevalier sans cheval, ça n'avait pas de sens commun ${ }^{29}$."

Que devint le cheval de M. de Montmagny ? Il paraît bien qu'on en a oublié le souvenir à peine quinze ans plus tard. Lorsqu'en 1663, Pierre Boucher fait rapport au roi des animaux domestiques que l'on trouve en Nouvelle-France, l'auteur s'exprime ainsi ${ }^{30}$ :

Voicy le nom de ceux (les animaux) que l'on amene de France, des Bœufs \& des Vaches; les bœufs servent a labourer la terre, \& à trainer du bois l'Hyver sur les neiges. Des Co-

26. Journal des jésuites, publié d'après le manuscrit original conservé aux archives du Séminaire de Québec. Par MM. les Abbés Laverdière, et Casgrain, 2e éd. (Montréal, 1893), 90.

27. Joseph Desjardins, Guide parlementaire historique de la province de Québec (Québec, 1902).

28. Pierre F.-X. de Charlevoix, Histoire et description generale de la NouvelleFrance, avec le journal historique d'Un Voyage fait par ordre du Roi dans l'Amérique septentrionale (3 vol., Paris, 1744), 1: 199.

29. Ernest Gagnon, Louis Jolliet (Montréal, 1913), 2e éd.

30. Pierre Boucher, Histoire Véritable et Naturelle des Mours et productions du pays de la Nouvelle France Vulgairement dite Canada (Paris, 1664), 64. 
chons en grand nombre: des Moutons il y en a peu: des Chiens, des Chats, \& des Rats. Voila les animaux que l'on nous a amené de France, qui font bonne fin en Ce Pay-icy.

On remarque que le gouverneur des Trois-Rivières cultive la minutie jusqu'à nous parler des rats. Il est bien permis de présumer qu'il ne se trouvait aucun cheval dans la colonie; sans quoi la plume d'un scribe aussi minutieux n'eût pas négligé d'en faire mention. En juillet 1665, voici qu'on débarque à Québec douze chevaux envoyés par le roi de France. On en a expédié vingt; huit sont morts durant la traversée.

L'arrivée du premier contingent de chevaux coinncide avec celle du célèbre régiment de Carignan. Nouvelle preuve que, par l'expédition de ce corps d'armée en Nouvelle-France, la Métropole visait un double but. Tracy voulait sans doute établir l'hégémonie française dans le pays des maraudeurs agniers; Talon, pour sa part, pensait surtout à rendre la colonie viable, à accélérer l'essor de son agriculture et d'abord de son peuplement et de son défrichement.

A ce sujet l'historien Charlevoix écrit ${ }^{31}$ :

Le reste du Régiment de Carignan, à quelques Compagnies pres, arriva avec M. de Salieres, qui en etoit Colonel, sur une Escadre, qui portoit aussi MM de Courcelle \& Talon, un grand nombre de Famille, quantite d'Artisans, des Engages, les premiers Chevaux, qu'on ait vus en Canada, des Bœufs, des Moutons, en un mot, une Colonie plus Considerable que Celle qu'on venoit renforcer.

Par contre la Relation de 1665 se montre plus précise relativement aux chevaux ${ }^{32}$ :

Le seizieme de Iuillet, (1665) arriva le navire du Havre, portant des chevaux, dont le Roy a dessein de fournir ce pais. Nos Sauvages, qui n'en avoient jamais veû, les admiroient, s'estonnans que les Orignaux de France (car c'est ainsi qu'ils les appellent), soient si traitables, et si souples à toutes les volontez de l'homme.

31. Pierre F.-X. de Charlevoix, Histoire et description générale de la NouvelleFrance, avec le journal historique d'Un Voyage fait par ordre du Roi dans l'Amérique. septentrionale (3 vol., Paris, 1744), 1: 381.

32. Relations des Jésuites contenant ce qui s'est passé de plus remarquable dans les missions des Pères de la Compagnie de Jésus dans la Nouvelle France. (3 vol., Québec, 1858),: 25. Voir relation de 1665 . 
Deux ans plus tard, en 1667, nouvel envoi de chevaux en Canada. Nous tenons le fait de la Mère Marie de l'Incarnation"33: "Sa Majesté a encore envoyé des chevaux, \& on nous a donné, pour notre part, deux belles juments et un cheval, tant pour la charrue que pour le charroi."

Et le roi de continuer dans ses bonnes dispositions envers l'agriculture de la colonie naissante. Le 16 février 1669 une ordonnance de 64,000 livres est émise pour le passage au Canada de 500 personnes des deux sexes, de douze cavales et de deux étalons ${ }^{34}$. Ces derniers solipèdes nous arrivent en 1670. Cette fois Sa Majesté fait preuve de plus de largesse. Ainsi en témoigne le sulpicien Faillon ${ }^{35}$ :

L'année 1670 , le Roj envoya pareillement un étalon \& douze juments, \& les fit distribuer aux gentilshommes du pays, les plus zélés pour la culture des terres: une jument à M. Talon, deux juments à M. de Chambly avec un étalon, une à M. de Sorel, une à M. de Contrecœur. une à M. de SaintOurs, une à M. de Varenne, deux juments à M. de Lachesnaye, une à M. de Latouche, une à M. de Répentigny, enfin la douzième à M. le Ber.

Toutes ces bêtes ont fait la traversée a bord du "Saint-JeanBaptiste" ${ }^{36}$. Un cheval a péri durant le trajet.

En France, Colbert s'intéresse toujours à la Nouvelle-France. A la fin de 1670 et au début de 1671 il émet des ordonnances relatives aux embarquements pour le Canada; il s'occupe particulièrement de l'achat, du passage et de lá nourriture des cavales et étalons destinés à la colonie laurentienne ${ }^{37}$.

Une fois rendus à destination, on ne confie pas ces dignes représentants de la race chevaline a quiconque sans exiger en retour certaines conditions. Ainsi les propriétaires doivent s'engager envers le roi à nourrir leurs chevaux pendant trois ans et si par leur faute

33. Lettres de Marie de l'Incarnation, (Clermont-Ferrand, 1857), 342.

34. Thomas Chapais, Jean Talon, intendant de la Nouvelle-France (1665-1672) (Québec, 1904), 340.

35. [ Abbe E.-M. Faillon ], Histoire de la Colonie française en Canada (3 vol.' Montréal, 1865-1866), 3: 222.

36. Thomas Chapais, Jean Talon, intendant de la Nouvelle-France (1665-1678) (Québec, 1904), 340.

37. Ibid., 340. 
un de ces animaux vient à mourir, le responsable versera deux cents livres d'amende au receveur du Roi. Par contre, les trois ans expirés, le propriétaire a le privilège de vendre sa bête, ainsi que les poulains qu'il a pu en avoir; mais là encore il a obligation de donner au receveur de Sa Majesté un poulain d'un an pour chaque cheval, ou la somme de cent livres. Il est par ailleurs convenu que lorsque ces poulains que le roi fait élever et nourrir seront parvenus à leur troisième année, leur distribution à d'autres particuliers s'effectuera toujours de la même manière ${ }^{38}$. Cette mesure ne doit pas étonner, si l'on se souvient que nous sommes à l'époque où Colbert s'occupe activement de reconstituer des haras en France. Rien de plus logique que de retrouver ici les mêmes soucis que dans la Métropole ${ }^{39}$.

Récapitulons. La Compagnie des Indes Occidentales, conformément aux instructions du ministre, fait régulièrement l'expédition de cavales et d'étalons pour le compte du roi. Les envois se chiffrent ainsi: en 1665, douze cavales, deux étalons; en 1667, encore douze cavales et deux étalons; en 1668, treize cavales et chevaux, soit au total quarante et un solipèdes pour une seule période de quatre ans. Ces chiffres peuvent paraitre modestes, mais il faut considérer que l'expédition de ces animaux est difficile et dispendieuse. En France une cavale vaut 120 livres, chaque étalon 200 livres. De plus le passage et la nourriture de ces bêtes en rendent le prix prohibitif. Ainsi en 1665, leur importation couta 11,200 livres au roi ${ }^{40}$. Malgré ces considérations le nombre des chevaux continue son ascension. Il en est envoyé quatorze en 1669 , treize en 1670 ; presque autant en 1671. De 1665 à 1672, le cheptel de la Nouvelle-France s'est enrichi d'environ quatre-vingts chevaux et cavales ${ }^{4 t}$.

On ne peut offrir de meilleures conditions de défrichement au colon et faire mieux pour l'expansion agricole. Aussi Colbert qui s'est tant dépensé pour l'avancement de l'industrie et de l'agricul-

38. Archives de la marine, volume Canada, tome II (1670-1676). En date du 20 aoat 1670 . 1919).

39. Charles de la Roncière, Un Grand Ministre de la Marine, Colbert, (Paris,

40. Thomas Chapais, Jean Talon, intendant de la Nouvelle-France (1665-1672) (Québec, 1904). 290.

41. Recensements du Canada 1665-1871 (Ottawa 1876), IV: recensements de 1692,1695 et 1698 . 
ture dans la colonie de la Nouvelle-France, écrira a Talon en date du 11 février $1671^{42}$ :

Je tiendrai la main à ce qu'il soit envoyé en Canada des cavales \& des ânesses, afin de multiplier ces expèces si nécessaires à la commodité des habitants.

Dans la distribution de 1670 nous remarquons que le Roi a pris soin de confier les chevaux à des gentilshommes terriens propriétaires de seigneuries déjà en culture. Cette attention n'est pas involontaire. On veut procurer à ces seigneurs des moyens nouveaux de travailler au défrichement et à l'ensemencement de leurs terres. L'on vise particulièrement à une plus grande production agricole. Les cavales canadiennes se montrent des plus prolifiques. Ainsi en témoigne la Relation de $1669^{43}$ :

De tous les animaux domestiques envoyés par le Roi dans la Nouvelle-France, les chevaux furent, en effet, ceux qui s'y multiplièrent le plus, quoique le nombre des autres y augmentât d'une manière étonnante.

Mais la statistique la plus sûre nous est fournie par le recensement général de la Nouvelle-France dressé durant l'été de 1681. Onze ans seulement après l'arrivée du contingent de 1670 , l'on compte 96 chevaux dans la colonie, issus pour la plupart des douze premiers sujets offerts par le Roi. Les villes de Québec avec 36 têtes et celle de Montréal avec 12 renferment à elles seules une grande partie de ces bêtes. Certaines régions rurales agricoles n'en possèdent encore aucune. Cet intéressant document de 1681 nous a conservé les noms des propriétaires de chevaux de l'époque. Il est opportun d'en donner ici la liste ${ }^{44}$.

42. Rapport de l'Archiviste de la Province de Québec pour 1980-1991 (Québec, 1931), 143. Voir correspondance échangée entre la cour de France et l'intendant Talon, en date du 11 février 1671.

43. Relations des Jesuites contenant ce qui s'est pass6 de plus remarquable dans les missions des Pères de la Compagnie de Jésus dans la Nouvelle-France (3 vol., Québec, 1858), 3: 3, relation de 1668.

44. Benjamin Sulte, Histoire des Canadiens-Français, 1608-1880 (8 vol., Montr6al. 1882-1884) 5: 53-88. 
Noms

Séminaire de Québec.

Couvent des Recollets (Québec)........

Couvent des Ursulines...............

Le Conseil Souverain.

Nicolas Dupont, sieur de Neuville.......

Paul Denys, sieur de Saint-Simon.......

Noel Levasseur.. . . . . . . . . . . . . . . . . . .

Thierry Delestre...................

Timothé Roussel, chirurgien...........

François Hurault. . . . . . . . . . . . . .

Joseph Renault...................

Noel Rose.... . . . . . . . . . . . . . . . .

Jean Poitras.....................

Jean Jobin. ......................

Mathieu Guay...................

Vincent Poirier dit Bellepoire.......... .

Vincent Beaumont...................

Jean Larchevêque.................. .

Marie Pelletier, veuve Denis Jean...... .

Rene Robineau, de Becandour......... .

Urbain Beaudry, taillandier...........

Bernard Despeche, de Repentigny. ..... .

Seminaire de Montréal. ..............

Congrégation Notre-Dame............

Robert Cavelier, de Montréal. . . . . . . . .

Jean Caillau.....................

Antoine Toupin, de Beaupré. . . . . . . . .

Guillaume Boucher, charron...........

Pierre Boucher, charron..............

Félix Aubert....................

Charles LeFrançois.................

Jacques Goullet..................

Rene Le Tartre..................

Guillaume Paget, taillandier...........

Nicolas Juchereau, de Beauport. ........

Charles Cadieu...................

Noel Langlois. . . . . . . . . . . . . . . . .

Toussaint Girou.................

Jacques Renault, de Charlesbourg.......

Pierre Vivier........................

Pierre Lefebvre.................

Jean Bernard, du village Saint-Joseph...

Pierre Guillebeault, du Petit Auvergne. .

Jean Janvier. . . . . . . . . . . . . . . . . .
Chevaux Juments

2

1

3

.6

3

1

1

1

5

2

1

2

1

1

1

2

3

2

1

2

3

5

4

2

3

1

1

2

1

2

1

2

1

1

1

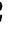

1

1

1

1

2


Noms

Louis Blanchard................. .

Pierre Lacroix..................

Michel Legardeur, de la côte St-Michel..

Pierre Maufort....................

Hubert Simon.....................

Ignace Bonhomme................

Jean Houde, de la côte Saint-Laurent. . .

TOTAL............
Chevaux Juments

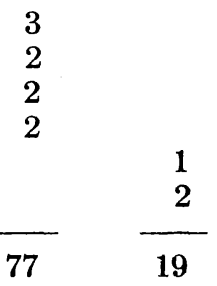

Malgré ce total assez imposant les représentants de la race chevaline en Canada resteront recherchés jusqu'à la fin du dixseptième siècle. Néanmoins dès février 1681 les habitants de la Martinique songent à importer des chevaux de l'Acadie ${ }^{45}$. En Nouvelle-France le nombre des chevaux ne progresse que lentement. Il passe de 156 en $1665^{46}$ à 175 l'année suivante ${ }^{47}$. Bientôt ces bêtes serviront à la petite armée canadienne. Lorsqu'en juillet 1696 les troupes se portent au pays des Onnontagués, monsieur de Callières monte "un cheval qu'il avoit faict venir sur des basteaux ${ }^{48}$."

Si le nombre de ces solipèdes est plutôt limité en terre laurentienne, il n'en est pas de même à pareille époque sur les bords du Mississipi. Nous lisons dans un écrit du chevalier de Tonti à la date du 22 avril $1685^{49}$ :

Ce que nous trouvâmes de particulier dans ces contrées, (passé la baie Saint-Louis) c'est que parmi le bétail à cornes, nous apperçûmes dans les prairies un grand nombre de Chevaux, mais si farouches, qu'on ne pouvoit les approcher.

Et le narrateur de continuer ${ }^{50}$ :

45. Collection de manuscrits contenant lettres, mémoires et autres documents historiques relatifs d la Nouvelle-France, recueillis aux archives de la Province de Québec ou copiés à l'étranger. (4 vol., Québec 1883-1885) I: 276.

46. Ibid. 1: 351 .

47. Ibid. 1: 390 .

48. Ibid. 1: 599 .

49. Dernières découvertes dans l'Amérique Septentrionale de $M$. de La Sale, mises au jour par M. le Chevalier Tonti, gouverneur du Fort Saint Louis, aux Illinois (Paris, 1697), 250.

50. Ibid.. 251. 
Le troisième jour (24 avril) nous trouvâmes sur le midi, quatre Cavaliers bottez.

Il s'agit de guerriers Queadis ou Mahis qui déjà savent admira* blement monter le cheval. Ces bêtes aperçues par l'ancien compagnon de la Salle descendent très probablement des quelques cavales et étalons abandonnés par De Soto plus d'un siècle et demi auparavant, alors que l'explorateur espagnol était à escarmoucher avec les bandes indiennes.

Lorsque M. de Meulles, intendant de la Nouvelle-France, vient visiter l'Acadie en 1685, il débarque dans la baie Verte pour trouver sur le rivage $\mathrm{M}$. de La Vallière qui l'attend avec des chevaux pour le conduire immédiatement au manoir de Beaubassin d'où il s'embarque sur la baie Française à destination de Port-Royal ${ }^{51}$. Les mentions de chevaux sont rares dans les annales acadiennes de l'époque. Aussi est-il difficile d'établir un nombre au moins approximatif de ces bêtes. Les premiers recensements relatifs à l'Acadie ne sont pas bien explicites à ce sujet. Pour la période allant de 1686 à 1693 ils nous indiquent simplement que "le betail a quintuple" ${ }^{2}$. Le cheptel du bassin des Mines est le plus beau de la péninsule. De cette dernière affirmation l'on peut conjecturer que la multiplication des chevaux et du bétail à cornes est corrélatif.

La Mothe-Cadillac nous fait en 1692 le portrait suivant du cheval acadien ${ }^{53}$ :

Les Chevaux (de l'Acadie) y sont de belle taille, bien traversés, forts, la jambe bonne, l'ongle dur, la teste un peu grosse, mais on ne prend point soin pour en elever a cause qu'on en trouve point le débit.

En 1696 "l'invasion" anglaise ravage de préférence la seigneurie de Beaubassin. Le cheptel ne se relève que difficilement de la razzia. Mais des documents et mémoires de La Mothe-Cadillac, Diéreville et autres nous apprennent qu'on le trouvait en bonne 'ssance dans tous les secteurs de l'Acad: $\sim 54$

51. Rameau de Saint-Père, Une Colonie féodale en Amerique - L'Acadie (1804 1881) (2 vol., Paris et Montréal, 1889), 1: 172.

52. Ibid., 220.

53. Ibid., 230. Archives de la marine.

54. Ibid., 236. 
Le cheval est plus recherché en Nouvelle-France. Il n'y est plus si rare, car il s'est bien multiplié. En 1692, vingt ans à peine après les premiers envois, il $y$ en a quatre cents dans la colonie; en 1695 , cinq cent quatre-vingts; en 1698 , six cent quatre vingtquatre ${ }^{55}$.

Dès lors l'on n'achète ni ne vend un cheval sans observer certaines formalités. Dans la plupart des cas, l'on se réservera les services d'un tabellion. Ainsi, le 10 aout 1699, par contrat passé devant le notaire Anthoine Adhémar, Jean Beaucham, de la Pointe-auxTrembles de Montréal, vend "une Cavalle agée de trois Ans sous poil Rouge, \& un cheval agé de trois ans sous poil Noir" ${ }^{56}$, au sieur Martinet dit Fonblanche, chirurgien de Ville-Marie. Et le scribe de signer avec paraple au bas de l'acte. La transaction est bâclée pour la somme de 450 livres cours du pays. Le sieur Fontblanche habite sur la rue Notre-Dame un terrain désigné au livre terrier sous la cote $165^{57}$.

On peut retracer bien d'autres actes notariés relatifs à l'achat et à la vente de chevaux entre divers habitants de la colonie. Voici quelques-unes de ces transactions:

Le 17 aout $1700^{58}$, vente par Charles Villiers marchand de Montréal à Laurent Renault également marchand du même lieu d'un cheval qui se trouve sur l'île Sainte-Hélène. Le vendeur s'engage a livrer la bête audit acheteur sur l'île en question pour le dimanche suivant moyennant la somme de 60 livres en argent et 10 cordes de bois de chauffage.

Le 16 octobre $1701^{59}$, vente par Françoise Moisan veuve d'Anthoine Brunet à Pierre Cardinal, de la côte Saint-Martin en l'île de Montréal, d'une cavale "sous poil de sourie" agée de 8 à 9 an s pour la somme de 100 livres et 20 minots de blé payables aux fêtes de la Chandeleur et de la Saint-Michel 1702.

55. Recensements du Canada 1665-1871 (Ottawa, 1876), IV; recensements de 1692,1695 et 1698.

56. Greffe d'Anthoine Adhemar (minute no 4809). En dépôt aux Archives judiciaires de Montréal.

57. Les Origines de Montréal (Mémoires de la Société Historique de Montréal, IIe livraison, Montréal, 1917), 162.

58. Greffe d'Antoine Adhemar (minute no 5272). En dépôt aux Archives judiciaires de Montréal.

59. Ibid., (minute no 5843). 
Le 20 avril $1704^{60}$, obligation par le sieur Seraphin Lauzon maitre-chaudronnier de Ville-Marie au sieur Charles Villiers de 24 cordes de bois de chauffage pour un cheval sous poil rouge.

Le 23 novembre $1704^{61}$, vente par Joseph Aubuchon, de SaintFrançois de l'île de Montréal à Jeanne-Margherite Le Noir épouse du sieur Louis Dusably co-seigneur de l'ile Dupas, d'une cavale sous poil brun agée de 5 ou 6 ans moyennant la somme de 100 livres.

Le 14 juin $1705^{62}$, vente par Jean Cailloud, de Montréal à Jean Lahaye habitant de la côte Saint-Laurent en la même île d'un cheval sous poil rouge agé de 4 ans avec son collier et sa bride pour la somme de 230 livres dont la moitié payable à la fête de Noel prochain et le reste à celle de l'année 1706 .

Le 20 août $1708^{63}, M$. Boucher vend un cheval au sieur Pierre You de la Découverte. Le 16 aout $1709^{64}$, l'acquéreur cède sa bête agée de 6 ans et sous poil rouge avec son harnais à Jacques Vaudry, charretier de Montréal. La transaction est bâclée pour la somme de 60 livres et 25 cordes de bois de chauffage. Le sieur You officier dans les troupes de la marine est l'un des principaux trafiquants installés à la Mission de l'île aux Tourtres (sic) près des Chenaux à Vaudreuil. Ce coin de terre est le berceau de la civilisation en terre vaudreuilloise. L'on peut conjecturer que le sieur de la Découverte y amena son cheval pour effectuer certains travaux à son "comptoir de traite". En l'occurrence ce serait le premier cheval des seigneuries de Vaudreuil et de Rigaud.

Le 3 avril 1712, vente par Pierre Plaisant, boucher de Montréal a Louis Roy du Sault-Saint-Louis d'un cheval "sous poil noir" 65 agé de 9 ans moyennant la somme de 25 livres payable à la fête de la Saint-Michel prochain.

Le 19 septembre $1713^{66}$, vente par Henry Catin et Jeanne Bros-

60. Greffe de Michel Lepallieur. En dépôt aux Archives judiciaires de Montréal.

61. Greffe d'Anthoine Adhemar (minute no 6973). En dépôt aux Archives judiciaires de Montréal.

62. Ibid., (minute no 7117).

63. Ibid., (minute no 8040).

64. Ibid., (minute no 8200).

65. Ibid., (minute no 8937).

66. Ibid., (minute no 9327). 
sard son épouse, de Montréal à Pierre Galien, charretier du même lieu d'un cheval sous poil noir agé de 8 à 9 ans et d'une cavale également de la même couleur agée de 3 ans avec trois harnois pour la somme de 300 livres, le labourage d'un champ et 30 cordes de bois de chauffage.

Le 2 novembre de la même année ${ }^{67}$, vente par Nicolas Bernard, de la rue Saint-Philippe à Montréal au sieur Lacharité, de la LonguePointe d'un cheval sous poil noir pour la somme de 80 livres dont la moitié payable le 2 décembre prochain et le reste à la fête de Noël.

Le 15 avril $1732^{68}$, obligation par François Bardet dit La Pierre au sieur Chaumont "Escrivain du Roy" de Montréal de 20 cordes de bois de chauffage pour le prix d'un cheval. Dix cordes devront être livrées pour janvier 1733 et le reste au cours du mois de février 1734.

Mais le document le plus typique en ce genre reste sans doute celui du louage d'un cheval en date du ler mai 1702. Par cette convention le sieur Charles Alavoyne, marchand de Montréal, loue un cheval sous poil gris blanc agé d'environ 5 ans à François Coron, habitant de la Rivière-des-Prairies. Le marché est conclu pour la durée d'un mois, à commencer du 2 mai courant pour se terminer au 2 juin suivant. Durant le temps du bail, le preneur s'oblige de nourrir, d'héberger et de prendre soin du cheval et s'il ne peut s'en servir pour labourer, il devra le ramener chez ledit sieur Alavoyne au plus tard dans huit jours. Le prix de location est de 22 livres. Une quittance inscrite au bas de l'acte indique que les clauses de "l'engagement" ont été respectées et qu'en outre, François Coron a payé 30 sols pour deux fers posés durant le temps du louage ${ }^{69}$.

Jusqu'ici le nombre des chevaux en Canada a été inférieur à celui de l'Acadie. Mais à la fin du dix-septième siècle la race chevaline canadienne prend une nouvelle expansion. De 684 qu'il est en 1698 , le total des chevaux passe à 1,872 en 1706. La progression devient si rapide que Pontchartrain s'en inquiète. Il craint que les Canadiens ne perdent leur précieuse qualité de marcheurs et qu'ils

67. Ibid., (minute no 9415).

68. Greffe de Pierre Raimbault. En dépôt aux Archives judiciaires de Montréal.

69. Greffe d'Anthoine Adhemar (minute no 6080). En dépôt aux Archives judiaires de Montréal. 
ne fassent plus usage des raquettes. Aussi le 10 juin 1710 recommandera-t-il au gouverneur "de tenir la main à la réduction des chevaux ${ }^{70}$."

D'autres fonctionnaires abondèrent dans le même sens. Le trop grand intérêt porté aux chevaux ne l'était, disait-on, qu'au préjudice des autres animaux de la ferme. D'où l'ordonnance de l'intendant Antoine Denis Raudot adressée aux habitants de Montréal en date du 13 juin $1709^{71}:^{\prime}$

Nous ordonnons que chaque habitant des côtes de ce gouvernement ne pourra avoir plus de deux chevaux ou cavales et un poulin, et ce, à commencer après les semences de l'année mil sept cent dix, leur donnant le dit temps pour pouvoir se défaire des chevaux qu'ils ont au-delà de ce nombre, et après lequel ils seront tenus de tuer ceux qu'ils auraient au-delà.

La multiplication des chevaux entraîne des inconvénients quant à la surveillance de ces bêtes. C'est ce qui expliquerait une autre ordonnance de Raudot en date du ler février 1706 qui défend à quiconque d'envoyer boire des chevaux "sans les conduire ou faire conduire par leurs. licols ou brides, et aux chartiers et voituriers de se tenir sur leurs charettes vuides en allant ou revenant à peine de prison et de dommages et interets des parties ${ }^{72}$."

Le 10 novembre 1716 , Jean-Baptiste Lacombe porte plainte contre Joseph Lacasse, Louis Téritet et le nommé Minot. Ce trio aurait renversé le plaignant avec leurs chevaux sur la route. A cet effet, l'intendant Raudot émet une nouvelle ordonnance ainsi rédigée ${ }^{73}$ :

Nous ('intendant) faisons defenses à toutes personnes, de quelques qualités et conditions qu'elles soient, de pousser leurs Chevaux contre les gens de pied qui seront dans leurs chemins, et, en cas d'accident arrivé aux dites gens de pied, nous condamnons Chacun des Contrevenants à dix livres l'amende applicable a celui qui aura été blessé, outre des dommages et interets qu'il pourra encourir contre lui.

70. Archives coloniales du Canada, mémoires 1706-1709, fol. 170.

71. Arrêts et réglements du Conseil Supérzeur de Québec et Ordonnances et Jugements des Intendants du Canada (3 vol., Québec, 1854-1856), 2: 273.

72. Ordonnances des Intendants et Arrêts du Consęl Supérieur de Québec (2 vol., Québec, 1803-1806) 2: 171 .

73. Complément des Ordonnances et Jugements des Gouverneurs et Intendants du Canada (3 vol., Québec, 1856) 3: 416. 
Le 28 juin 1710, il y a déjà eu ordonnance du même intendant pour défendre aux habitants de Verchères et de toutes les autres côtes du gouvernement de Montréal de laisser aller leurs chevaux et poulains dans les blés à peine de dix livres d'amende et "qui enjoint de faire enferger leurs dites chevaux et poulains ${ }^{74}$."

Le gouverneur M. de Vaudreuil et l'intendant Michel Bégon, dans un mémoire adressé au Ministre en date du 12 novembre 1712, soulignent que le Sieur Daigremont "a exhorté autant qu'il a pa les habitans de la Colonie à diminuer le nombre de leurs chevaux qui consomment pendant L'hiver la plus grande partie de leur fourrage"75. Pour solutionner ce problème Bégon demande au Ministre qu'on lui confère les mêmes pouvoirs déjà accordés à son prédécesseur Raudot, quitte à rendre compte au roi de son administration en ce sens à la fin de chaque année. L'intendant soutient que c'est le seul moyen d'augmenter les bêtes à cornes et les moutons, car, à cause de la rareté de ces dernières bêtes, le prix de la viande est haussé du tiers depuis l'an passé. Et Vaudreuil et Bégon de faire chorus $^{76}$ :

la diminution du nombre des chevaux pourra obliger les habitans de la Colonie a aller plus souvent en raquettes, [ nous ] les y porteront autant que faire ce pourra, estants persuadez comme vous Monseigneur, qu'il est nécessaire de les entretenir dans cet usage qui leur donnera toujours de la superiorité sur les anglois.

Plus loin les administrateurs reprennent le même refrain ${ }^{77}$ :

Il faut absolument diminuer le nombre des chevaux et remettre les habitans a aller en raquette. Il est de l'interest de ceux qui sont en teste de la colonie que les habitans soient forts et robuste.

Dans une lettre adressée au Conseil de la Marine, le 26 février 1717, MM. Vaudreuil et Bégon reconnaissent qu'il y a 3,786 che-

74. Inventaire des Ordonnances des Intendants de la Nouvelle-France conservées aux Archives de la province de Québec (4 vol., Beauceville, 1919), 1: 104.

75. Rapport de l'Archiviste de la province de Quebec pour 1947-1948 (Québec, 1948), 176. Correspondance entre M. de Vaudreuil et la Cour, 12 9bre 1712.

76. Ibid.

77. Ibid. 
vaux en Canada ${ }^{78}$. La seule objection sérieuse à la diminution de ces bêtes, on le reconnait, c'est le voiturage d'hiver. Mais le problème est vite résolu. Que ne se sert-on des bœufs comme aux premières heures de la colonie laurentienne ? Ou encore que ne chausse-ton tout simplement ses raquettes ${ }^{79}$ ?

Malgré ces "bonnes dispositions" il devient impossible d'enrayer la multiplication des solipèdes. L'augmentation constante des chevaux continue de troubler le gouverneur de la colonie et surtout les autorités de la métropole. A-t-on réussi à contenir cette augmentation? Quci qu'il en soit, le chiffre des chevaux devient subitement stationnaire: 5,063 en 1720 et 5,056 en $1734^{80}$. Pourtant gouverneurs et intendants trouvent le nombre encore trop élevé. On s'inquiète de voir les fils d'habitants mettre toute leur ambition à élever les meilleurs chevaux de selle ${ }^{81}$. Beauharnois et Hocquart s'accordent avec Vaudreuil à ce sujet. Il faut absolument empêcher la jeunesse canadienne de perdre le gout des marches. Alors que faire du surplus de chevaux? On ne peut pourtant pas les sacrifier. Il ne reste plus qu'à les vendre hors de la Colonie. D'autant plus “qu'ils sont de bonne qualité, forts et résistants à la fatigue" 82 . Dès le 29 avril 1727 l'on propose d'en exporter aux Antilles ${ }^{83}$. De Marly, le 8 mai 1731, Sa Majesté informe le marquis de Beauharnois de la satisfaction qu'elle a "d'apprendre que les négociants du Canada commencent enfin à sentir les avantages qu'ils y aurait de transporter aux Isles des chevaux et des bestiaux" ${ }^{84}$. Le 24 mai 1728, c'est au tour du président du conseil de la marine d'écrire à l'intendant Dupuy

78. Documents Historiques, Correspondance échangée entre les Autorités françaises et les gouverneurs \& intendants (Québec, 1893), 128.

79. Rapport de l'Archiviste de la province de Québec pour 1947-1948 (Québec, 1948), 176. Correspondance entre M. de Vaudreuil et la Cour, 12 9bre 1712. et 57 .

80. Recensement du Canada 1665-1871 (5 vol., Ottawa, 1876), IV: 28, 52, 53

81. Emile Salone, La Colonisation de la Nouvelle-France - Etude sur les origines de la nation canadienne-française (Paris, s.d.), 381.

82. Franquet, Voyages et mémoires sur le Canada (Québec, Institut Canadien de Québec, 1889), 27.

83. Collection de manuscrits contenant lettres, mémoires, et autres document8 historiques relatifs $\grave{a}$ la Nouvelle-France, recueillis aux archives de la Province de Québec, ou copiés à l'étranger. (4 vol., Québec, 1883-1885), 3: 130.

84. Ibid., 3: 131. 
pour lui faire savoir qu'il souhaiterait que le Canada fasse le commerce des chevaux avec les Iles, à condition que tout soit laissé à l'initiative privée. Les Anglais de la Nouvelle-Angleterre vendent déjà de ces bêtes aux Antilles pour une somme variant entre 300 à 400 livres chacune ${ }^{84^{\mathrm{a}}}$. L'année suivante, le $2 \mathrm{mai}$, nouvelle missive du président du conseil à Hocquart l'informant qu'il n'approuve pas la proposition déjà faite par l'intendant Dupuy pour l'établissement de haras en Canada. L'hiver est trop long et il vaut mieux diminuer le nombre de ces solipèdes. L'élevage des bestiaux est plus profitable. On pourrait les convertir en salaisons pour les Isles, ce qui serait beaucoup plus pratique que de transporter des chevaux ${ }^{84^{\mathrm{b}}}$. Le 17 avril 1731, nouvelle instruction du même au même: "Il peut être difficile d'établir le commerce des chevaux avec la Martinique, mais il ne faut pas se rebuter" $"{ }^{\mathrm{c}}{ }^{\mathrm{c}}$. Mais le président du conseil de marine est persévérant. De Marly le 22 mai 1731, il écrit à MM. Beauharnois et Hocquart pour leur suggérer d'imposer un droit annuel d'un minot de blé par cheval ${ }^{84^{d}}$. Le 22 avril 1732 le roi demande à M. de Beauharnois s'il ne serait pas opportun d'expédier le surplus des chevaux canadiens à l'Ile Royale ${ }^{84^{\mathrm{e}}}$. Enfin le $29 \mathrm{du}$ même mois, le président du conseil informe MM. Beauharnois et Hocquart que sur leurs représentations des difficultés qu'entraînerait la levée de l'imposition proposée d'un minot de blé par an pour chaque cheval que les habitants auraient, le roi s'est décidé à ne pas ordonner cette taxe. N'empêche qu'ils doivent toujours diriger leurs efforts en vue de diminuer le nombre des chevaux et augmenter celui des bestiaux ${ }^{84^{f}}$. Pour encourager cette mesure Hocquart croit trouver une solution en proposant de n'autoriser qu'un cheval pour quatre bœufs à tous les habitants de la Nouvelle-France ${ }^{85}$. Vaine

84a. Rapport concernant les Archives Canadiennes pour l'année 1904 (Ottawa, 1905), Appendice K, Sommaire des Documents à Paris fait par feu M. Edouard Richard, 101.

84b. Ibid., 117.

84c. Ibid.. 139.

84d. Ibid. 144.

84e. Ibid., 157.

84f. Ibid., 159.

85. Hocquart au ministre, 4 octobre 1731, Archives coloniales du Canada, 1731, folio 170 . 
tentative. Dans un mémoire daté du 26 décembre 1734 et envoyé de Québec au roi, MM. de Beauharnois et Hocquart avouent que l'échec est complet ${ }^{86}$ :

Nous n'avons pas encore déterminé les armateurs du Canada à entamer le commerce des chevaux pour les isles (les Antilles).

L'exiguïté des bâtiments du pays et l'étendue que chaque cheval occupe avec le fourrage et l'eau qui lui sont nécessaires durant une traversée de deux mois, sont de grands obstacles à ce commerce, sans compter les risques de mort auxquels les chevaux sont exposés dans un vaisseau où ils sont mal soignés et placés constamment debout. De plus le prix en devient prohibitif. A Québec en 1734 un cheval coute de 100 à 150 francs. Le transport d'un seul, à bord, avec son fourrage et trois tonneaux d'eau, conterait de 300 à 400 francs, de sorte que, rendu aux "Isles", un cheval reviendrait a la jolie somme de 500 francs $^{87}$.

Le 20 janvier 1742, ordonnance de M. de Monrepos défendant de faire galoper les chevaux dans les rues de Montréal. Le document faisait aussi défense d'envoyer boire les chevaux à l'abreuvoir public sans les y conduire avec un licol. Lorsqu'on attellera deux bêtes à une voiture il faudra "mettre des guides au cheval de devant, comme à celui de derrière, afin de pouvoir les arrêter l'un et l'autre à l'ins$\operatorname{tant}{ }^{88} . "$

Le savant naturaliste suédois, Peter Kalm, lors d'un voyage d'étude en Canada à la fin du régime français, note en juillet $\mathbf{1 7 4 9}$ qu'on a l'habitude de laisser les chevaux dehors pendant l'hiver au fort Saint-Frédéric. Ces bêtes trouvent leur pâture dans les bois et se nourrissent $d$ 'herbes sèches ${ }^{89}$. Plus loin l'auteur souligne la rareté

86. Collection de manuscrits contenant lettres, mémoires, et autres documents historiques relatifs a la Nouvelle-France, recueillis aux archives de la Province de Québec, ou copiés à l'étranger (4 vol., Québec, 1883-1885), 3: 170.

87. Ibid., 3: 170.

88. Repertoire des Arrets, Edits, Mandements, Ordonnances et Reglements relatifs d Montrél sous le Régime Français (1640-1760), (Montréal, 1919): 103.

89. "Voyages de Kalm en Amérique", Mémoires de la Société Historique de Montréal, 8ème livraison, (Montréal, 1880), 12.

90. Ibid., 38. 
de ces solipèdes à Saint-Jean ${ }^{90}$. Et Kalm de reprendre l'éloge du cheval, à l'instar de La Mothe-Cadillac un demi-siècle plus tôt ${ }^{91}$ :

Tous les chevaux canadiens sont forts, vifs, bien faits. Les habitants ont la coutume de couper la queue à leurs chevaux, ce qui est une vraie cruauté ici, puisqu'on les prive ainsi de leur unique moyen de défense contre les moucherons et les taons. Cette coutume vient peut-être de ce qu'ils attellent leurs chevaux l'un devant l'autre; et pour empêcher celui de devant de blesser les yeux de celui qui est dans les timons en agitant sa queue, ils auront pris le parti d'écourter tous leurs chevaux.

Enfin le Suédois note le prix: "Un cheval de moyenne encolure coute maintenant quarante francs et plus. Un beau cheval vaut cent francs ${ }^{92}$."

L'endurance du cheval canadien sur la route sait procurer d'heureux moments à ses propriétaires. Les officiers et les hauts fonctionnaires organisent des excursions pour vaquer aux besoins de l'administration. Ces randonnées sont souvent agrémentées par la présence du beau sexe. La visite de l'ingénieur Franquet à Oka, du 27 au 29 février 1752, pour y faire l'inspection de la place fortifiée, est restée l'exemple le plus typique de ces promenades où l'on savait joindre l'utile à l'agréable. Une caravane de carrioles tirées par des chevaux fringants a traîné l'ingénieur français et sa suite joyeuse durant toute la durée du voyage ${ }^{93}$.

Lorsque à son tour le chevalier de Raymond fait en 1754 la tournée des postes armés du Canada, il précise que "la nourriture des chevaux et l'entretien des harnois et Charrette" dans les places et forts de Frontenac, Toronto, Niagara, Belle-Rivière et Détroit sont à frais communs entre le roi et les négociants ${ }^{94}$.

On peut conjecturer que l'ordonnance de Monrepos en date du 20 janvier 1742 ne fut guère observée des habitants puisqu'une

91. Ibid., 142.

92. Ibid., 229.

93. Franquet, Voyages et Mémoires sur le Canada (Québec, Institut Canadien de Québec, 1889), 189.

94. Rapport de l'Archiviste de la Province de Québec pour 1927-1928 (Québec), 1928). Mémoire sur les postes du Canada adressé a $M$. de Surlaville en 1754, par le Chevalier de Ravmond. 
décade plus tard, soit le 23 décembre 1752 , le même intendant émet une nouvelle ordonnance semblable à la première ${ }^{95}$.

Vers la même époque en Acadie, Lawrence se montre moins scrupuleux que le chevalier de Raymond et dans l'acquisition et dans l'entretien de ces bêtes. Ce triste sire est un amateur passionné de chevaux. Il n'en fixe pas la valeur comme Kalm quelques années auparavant. Il ne se donne même pas la peine de maquignonner. Le gouverneur use de moyens plus radicaux. Il fait tout simplement visiter les écuries des Acadiens par ses seconds qui font la sélection sur place des plus beaux sujets et les lui ramènent sans autre formalité. Son principal agent dans ces razzias "légalisées" est Moïse de Les Derniers, un Suisse huguenot, apparenté sans doute à ses homonymes que l'on retrouve quelques décades plus tard dans le haut de la seigneurie de Vaudreuil sur les bords du lac des DeuxMontagnes. C'est sur les instructions de Lawrence que, du fort Edward, le 3 septembre 1755 Murray donne le sauf-conduit suivant ${ }^{96}$ :

Permit the Bearer Moses de les Derniers to go to Grand Pré, to the Rivers Cannard and Habitant to look for some horses for the use of the lieutenant governor and bring the same to this fort.

Muni de cette permission, l'émissaire de Murray parcourt toutes les paroisses de cette région acadienne, pour le compte de Lawrence, et fait partout le sac des écuries. Soulignons que le sauf-conduit donné à Les Derniers, pour pratiquer son singulier commerce, est daté du 3 septembre, soit l'avant-veille de l'holocauste de Grand-Pré. Lawrence éprouve de véritables faiblesses pour les beaux chevaux. Il veut être parmi les premiers à se partager les dépouilles des Acadiens.

De son côté Murray, sous prétexte d'obéir aux ordres de Lawrence, n'hésitera pas à piller les haras acadiens. Il écrit à Winslow que la plupart des chevaux qu'il a vus ne plairont pas a Lawrence. Mais il a été informé, le même jour, qu'un cheval noir appartenant à Armand Gros, de Grand-Pré, ferait un excellent cheval de selle

95. Repertoire des Arrets, Edits, Mandements, Ordonnances et Reglements relatifs d Montréal sous le Régime Français (1640-1760), (Montréal, 1919), 125.

96. Abbé H.R. Casgrain, Un pèlerinage au Pays d'Evangéline (Québec, 1888), 179. 
parfaitement au goût de Lawrence. Et Murray de demander à Winslow d'ordonner à René Le Blanc fils et à quelques autres Acadiens d'aller s'en emparer pour l'amener au sieur Lawrence ${ }^{97}$.

Le 19 septembre 1755 Winslow informe Lawrence qu'il vient de rafler 500 chevaux aux Acadiens et qu'il garde ces bêtes aux casernes ${ }^{98}$. A la fin du "Grand Dérangement" le bilan des chevaux ainsi volés s'élève à plus de 10,000 têtes d'après le duc de Nivernois ${ }^{99}$. Lawrence, Winslow, Murray, Moïse de Les Derniers et sa bande, ainsi que le commissaire Saul, se sont partagé ces dépouilles ${ }^{100}$.

Dans la colonie laurentienne le cheval sera bientôt sacrifié aux nécessités de la guerre. L'état d'alerte où vit continuellement la Nouvelle-France depuis 1755 a dégarni les champs. Les hommes ont été dirigés vers les points menacés des frontières. Les vivres deviennent de plus en plus rares. Le 2 décembre 1757, le marquis de Montcalm écrit de Québec au chevalier de Lévis ${ }^{101}$ :

On va donner du cheval à nos troupes. Monsieur l'intendant vouloit une distribution toute en boeuf et une autre toute en cheval. Nous avons obtenu qu'on donneroit à chaque distribution moitié l'un moitié l'autre, et M. Cadet m'a dit écrire les mêmes choses pour Montréal.

A Québec les troupes se prêtent de bonne grâce à cette nouvelle nourriture. Mais la garnison du fort de Chambly se montre plus récalcitrante. Elle est loin de trouver succulent le nouvel aliment. De Québec le 4 décembre (1757) Montcalm adresse à Lévis toute une liste de plats préparés avec de la viande de cheval. Le commandant des troupes termine sur ce ton ${ }^{102}$ :

on mange chez moi du cheval de toutes façons, hors la soupe.

Mais il semble que tous les officiers supérieurs ne prêchent pas ainsi par l'exemple. A Montréal en particulier ils répugnent à man-

97. Journal de Winslow, 108.

98. Emile Lauvrière, La Tragédie d'un Peuple - Histoire du peuple acadien (2 vol., Paris, 1923), 1: 493.

99. Abbé H.R. Casgrain, Un Pèlerinage au Pays :d'Evangéline (Québec, 1888), 539. Lettre du duc de Nivernois sur la dispersion des Acadiens, 2 déc., 1762.

100. Rameau de St-Père, Une Colonie Fédale en Amérique - L'Acadie (16041881) (2 vol., Paris et Montréal. 1889) 2: 166.

101. Lettres du marquis de Montcalm, collection Lévis, (Québec, 1894), 86.

102. Ibid., 89. 
ger du cheval. Une lettre de Montcalm en date du 16 décembre de la même année en témoigne ${ }^{103}$ :

Le soir (les soldats du régiment de la Reine) mettent cuire le cheval, l'écument bien, jettent la première eau, le retirent, en font le lendemain de la bonne soupe en le remettant au pot avec le bœuf, mange le bœuf qui a servi à faire la soupe bouillie le matin, et le soir le cheval en frigousse. La colonie fait de même. Mais à Montréal Béarn (le régiment) est chez l'habitant, avec qui il vit et qui montre de la répugnance pour cette nourriture. Il faudroit pour le détr jire que le général, vous, (le chevalier de Lévis) MM. Martel, Deschambault et tous les gens aisés fussiez sur le ton d'en manger; vos domestiques en mangeroient et, de là, cela viendroit à la mode parmi le peuple.

Malgré ces "saignées" la race chevaline canadienne reste nombreuse après la capitulation de Montréal en septembre 1760. Un an avant le traité de Paris qui scelle définitivement le sort de la Nouvelle-France, le total des chevaux en Canada s'élève à plus de 15,000. Le recensement du gouvernement de Québec dressé en 1762 nous indique 4,474 chevaux pour la région ${ }^{104}$. Celui des gouvernements de Montréal et de Trois-Rivières rédigé en 1765 fixe à 13,757 le nombre des chevaux dans ce secteur ${ }^{105}$. Dans la seule seigneurie de Vaudreuil qui vient de s'ouvrir au défrichement, on en compte déjà $66^{106}$.

Rigaud de Vaudreuil, Robert-Lionel Seguin, L.S.S. ce 14 juin 1951 .

103. Ibid., 93.

104. Rapport de l'Archiviste de la Province de Québec pour 1925-1926 (Québec, 1926). Recensement du Gouvernement de Québec en 1762.

105. Rapport de l'Archiviste de la Province de Québec pour 1986-1987 (Québec, 1937). Recensements des gouvernements de Montréal et de Trois-Rivières en 1765.

106. Ibid. 